\title{
Link Quality Estimation with the Gilbert-Elliot Model for Wireless Sensor Networks
}

\author{
Ana Bildea*, Olivier Alphand, Franck Rousseau, and Andrzej Duda \\ University Grenoble Alps, Grenoble Institute of Technology, CNRS Grenoble Informatics Laboratory, Grenoble, France \\ Email: \{firstname.lastname\}@imag.fr
}

\begin{abstract}
In this paper, we apply the Gilbert-Elliot model to analyze measurements on a large-scale wireless sensor testbed with nodes equipped with $\mathrm{CC1101}$ and $\mathrm{CC2420}$ radio chips. The model considers a packet loss process as a sequence of transitions between Good and Bad states. We analyze the Packet Reception Ratio (PRR) based on the probabilities derived from the GilbertElliot model. We show that the probabilities and other parameters (run and loss lengths) can very well discriminate between the main categories of link quality such as good and weak links. Identification of link quality is crucial for routing protocols such as RPL and LOADng.
\end{abstract}

Keywords-Gilbert-Elliot Model, Link Quality Indicator (LQI), link and routing metrics, Wireless Sensor Networks, IoT-LAB

\section{INTRODUCTION}

In this paper, we present an approach based on the GilbertElliot model at the packet level to analyze the Packet Reception Ratio (PRR) and to find the best way to discriminate good links from weak ones. Recent work on routing protocols emphasized the importance of using stable metrics of link quality (one metric used with RPL is ETX that depends on PRR [1] and LOADng uses the number of weak links in a path as a metric [2]). This paper complements our previous work on characterizing the quality of wireless links in wireless sensor networks [3].

The transmission quality in terms of the Packet Reception Ratio (PRR) depends on the received signal strength, the level of interference, and the ability of the receiver to correctly decode transmitted information. The RSSI (Received Signal Strength Indicator) indicator provides an estimate of the signal energy at the receiver. LQI (Link Quality Indicator) may provide a better correlation with PRR than RSSI. LQI for $\mathrm{CC} 1101$ gives "an estimate of how easily a received signal can be demodulated by accumulating the magnitude of the error between ideal constellations and the received signal over the 64 symbols immediately following the sync word" [4].

In the previous work, we reported results of measurements on SensLAB (now IoT-LAB) [5], an indoor wireless sensor network testbed with a large number of nodes equipped with CC1101 and CC2420 radio chip (IoT-LAB is the current name of the platform [6]).

In this paper, we apply the Gilbert-Elliot model to analyze PRR based on measurements on IoT-LAB. We consider the packet reception process as a sequence of bits: 1 stands for a successful packet reception whereas 0 denotes a lost or corrupted packet. Such formulation leads to the possibility of

*Ana Bildea is now with Arago Systems, Sophia Antopolis, France. computing the stationary probabilities of being in the Good or Bad state in the Gilbert-Elliot model. We show that the probabilities and other parameters (run and loss lengths) can discriminate fairly well between links of different quality. We also apply the same approach to LQI by transforming a LQI value to a $0-1$ process and computing the Gilbert-Elliot probabilities. Similarly, the resulting probabilities lead to good discrimination of quality for intermediate links.

\section{EXPERIMENTAL SET UP}

We recall the experimental set up reported in the previous work [3]: we have run experiments on the IoT-LAB platform available in Strasbourg composed of 240 WSN430 nodes with the $\mathrm{CC} 1101$ radio distributed across three trays at different heights. Each tray contains 80 nodes arranged in a regular grid (10x8) with a distance between contiguous nodes of about $1 \mathrm{~m}$. A node is composed of a MSP430F1611 CPU (48KB ROM, $10 \mathrm{~KB}$ RAM) and a $\mathrm{CC} 1101$ radio operating at $868 \mathrm{MHz}$. Its transmission power ranges between $-30 \mathrm{dBm}$ and $10 \mathrm{dBm}$, and the reception sensitivity is set to $-88 \mathrm{dBm}$.

In a single experiment, we use one tray at a time, i.e. 80 nodes. We observe the quality of transmission of a node that broadcasts a total of 5000 packets of 110 bytes every $0.5 \mathrm{~s}$. There is no other ongoing transmissions so there is no interference nor contention between nodes. When one node broadcasts its packet, the other 79 nodes are active and ready to receive-they log the values of LQI and RSSI of the received packet. The values are recorded for the correctly received packets with good CRC and also for those with incorrect CRC. As there is one sender at a time, we are able to relate the sender and the receiver of a packet even if the receiver cannot decode a packet.

The receiver nodes do not acknowledge frames and the MAC layer does not retransmit frames in case of failed transmissions. After the experiment, we compute for each link: i) the average value of RSSI over all received packets, ii) the average value and the standard deviation of LQI, iii) the average value of the Packet Reception Ratio (PRR) of each link as the proportion between the number of correctly received packets (correct CRC) to the total number of sent packets. The observed values of RSSI only slightly varied, so we have not analyzed the standard deviation of RSSI.

We assume that all nodes can potentially communicate with each other so that the number of unidirectional links is 6320 (80 sender nodes times 79 receiver nodes). We run the experiments with two levels of the transmission power: $0 \mathrm{dBm}$ and $-10 \mathrm{dBm}$. The bit rate is $60 \mathrm{~kb} / \mathrm{s}$ and nodes use the 
2FSK modulation. Table I summarizes the parameters of the experiments.

TABLE I. EXPERIMENT PARAMETERS FOR CC1101

\begin{tabular}{ll}
\hline Experiment area & $10 \mathrm{~m} \times 8 \mathrm{~m} \times 2 \mathrm{~m}$ \\
\hline Number of nodes & $3 \times 80$ \\
\hline Traffic type, interpacket interval & broadcast, $100 \mathrm{~ms}$ \\
\hline Number of sent packets & 5000 \\
\hline Packet size & 110 bytes \\
\hline Transmission power & $0 \mathrm{dBm},-10 \mathrm{dBm}$ \\
\hline Topology & grid \\
\hline
\end{tabular}

For experiments with the CC2420 radio, we have used the IoT-LAB platform in Lille composed of 256 WSN430 nodes scattered across two horizontal trays of different heights and one vertical tray. Each of the horizontal tray contains 100 nodes arranged in a grid of $20 \times 5$ with a distance of about $0.6 \mathrm{~m}$ between nodes. A node has a CC2420 radio chip operating at the $2.4 \mathrm{GHz}$ band.

Table II shows the parameters of the experiments. We use for our study one tray of 100 nodes. The bit rate is $250 \mathrm{~kb} / \mathrm{s}$ and nodes use the OQPSK modulation. The measurement process of packet reception was the same as on the CC1101 platform.

TABLE II. EXPERIMENT PARAMETERS FOR CC2420

\begin{tabular}{ll}
\hline Experiment area & $11.4 \mathrm{~m} \mathrm{x} 2.4 \mathrm{~m}$ \\
\hline Number of nodes & $20 \times 5$ \\
\hline Traffic type, interpacket interval & broadcast, $100 \mathrm{~ms}$ \\
\hline Number of sent packets & 1000 \\
\hline Packet size & 110 bytes \\
\hline Transmission power & $0 \mathrm{dBm},-10 \mathrm{dBm}$ \\
\hline Topology & grid \\
\hline
\end{tabular}

\section{LINK CHARACTERIZATION}

\section{A. Categories of Link Quality}

We consider three main categories of link quality: good links with $P R R \geq 80 \%$, intermediate with $20 \% \leq P R R<$ $80 \%$, and bad ones with $0<P R R<20 \%$. Such categories appeared in previous studies [7], [8], but other thresholds are also possible, e.g. $90 \%-10 \%$.

Table III gives the proportion of links in each category (over all 6320 unidirectional links) for the CC1101 platform. In addition to the categories, we provide the proportion of the links with $P R R=0 \%$ (actually, no link between nodes).

TABLE III. PROPORTION OF LINKS IN EACH CATEGORY

\begin{tabular}{ccccc}
\hline Transmission power & good & intermediate & bad & $P R R=0 \%$ \\
\hline 0dBm & $49 \%$ & $8 \%$ & $10 \%$ & $33 \%$ \\
\hline $\mathbf{- 1 0 d B m}$ & $44 \%$ & $6 \%$ & $9 \%$ & $41 \%$ \\
\hline
\end{tabular}

We can observe that roughly half of links are good, a large number of links are bad and decreasing the transmission power to $-10 \mathrm{dBm}$ only slightly affects the proportion of good links. The number of intermediate links is fairly low.

\section{B. Gilbert-Elliot Model}

In this part, we apply the Gilbert-Elliot model (GE), a 2state Markov model, to packet reception sequences to analyse PRR.

The GE model is widely used to represent the state of a channel ( $\mathrm{G}$ - Good, B - Bad) by analyzing the errors on the

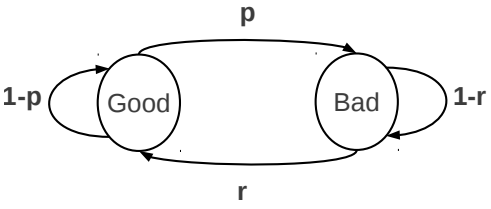

Fig. 1. Transition probability Gilbert-Elliot: 2-state Markov diagram. Good state represents a better quality channel whereas Bad state corresponds to a lower quality channel.

channel [9] (cf. Figure 1). The model has two states, the Good state corresponds to a successful reception and the Bad state to a loss. It was also used in communication networks to capture the temporal correlation of packet losses [10]. To study PRR using the model, we consider the packet reception process as a sequence of bits: 1 stands for a successful packet reception whereas 0 denotes a lost or corrupted packet.

The GE model is defined by the transition matrix $M$ between states $s_{t}$ at time $t$ :

$$
M=\left(\begin{array}{cc}
1-p & p \\
r & 1-r
\end{array}\right)
$$

where $p=P\left(s_{t}=B \mid s_{t-1}=G\right)$, and $r=P\left(s_{t}=G \mid s_{t-1}=\right.$ $B), 0<p<1$ and $0<r<1$ are the transition probabilities between states, respectively.

A transition from $G$ to $B$ takes place whenever the current packet is lost when the previous one was successful. In the opposite direction, a transition from $B$ to $G$ state takes place whenever the current packet is successfully received, but the previous one was lost. The conditional loss probability of remaining in the good state (probability that a loss arises after a success) is given by $p$ while the conditional loss probability remaining in the bad state is denoted by $1-r$ (probability that a loss arises after a loss).

The stationary probability of the Good state $\pi_{G}$ and the Bad state $\pi_{B}$ are given by:

$$
\pi_{G}=\frac{r}{p+r}, \quad \pi_{B}=\frac{p}{p+r}
$$

Considering the given stationary state probabilities, the loss probability is defined as:

$$
\pi_{l o s s}=p * \pi_{G}+(1-r) * \pi_{B}=\pi_{B}
$$

Parameter $\mu$ called channel memory is defined as:

$$
\mu=1-p-r,-1 \leq \mu \leq 1
$$

When $\mu=0$, the packet loss process is memory-less. $\mu$ becomes negative for oscillatory links.

To show how the parameters of the GE model can be obtained from packet loss observations, let us consider that the first link has sequence $_{1}=[011110]$ (1 denotes a received packet, 0 denotes a lost packet). The second link is characterized by sequence $_{2}=[101011]$. We see that sequence $_{1}$ has 4 consecutive successful packet receptions whereas sequence 2 records only 2 . 
Transition matrices $M_{1}\left(p_{1}, r_{1}\right), M_{2}\left(p_{2}, r_{2}\right)$ for sequence 1 and 2 are the following:

$$
M_{1}=\left(\begin{array}{cc}
0.75 & 0.25 \\
1 & 0
\end{array}\right) \quad M_{2}=\left(\begin{array}{cc}
0.33 & 0.66 \\
1 & 0
\end{array}\right)
$$

We obtain the following transition probabilities: the first link has $p_{1}=0.25$ and $r_{1}=1$, and the second link has $p_{2}=0.66$ and $r_{2}=1$.

TABLE IV. PERFORMANCE FOR Link $k_{1}$ AND Link $_{2}$.

\begin{tabular}{|c|c|c|c|c|c|c|c|}
\hline Link & $P R R$ & $p$ & $r$ & $\pi_{G}$ & $\pi_{B}$ & $\pi_{\text {loss }}$ & $\mu$ \\
\hline $\mathbf{1}$ & 0.67 & 0.25 & 1 & 0.8 & 0.2 & 0.2 & -0.25 \\
\hline $\mathbf{2}$ & 0.67 & 0.66 & 1 & 0.6 & 0.39 & 0.39 & -0.66 \\
\hline
\end{tabular}

Table IV summarizes the performance derived from the GE model applied to $\operatorname{Link}_{1}$ and $\operatorname{Link}_{2}$. Moreover, even if both links have a the same $P R R=0.67(67 \%)$, Link $_{1}$ would be preferred over $\operatorname{Link}_{2}$ as it records a higher good stationary probability ( $\pi_{G}$ of 0.8 compared to 0.6 ) and a higher negative channel memory $\left(\mu=-0.25\right.$ indicating that Link $_{1}$ is less oscillatory than Link $_{2}$ with $\left.\mu=-0.66\right)$.

Our goal is to use the stationary probabilities given by the GE model to estimate the quality of links.

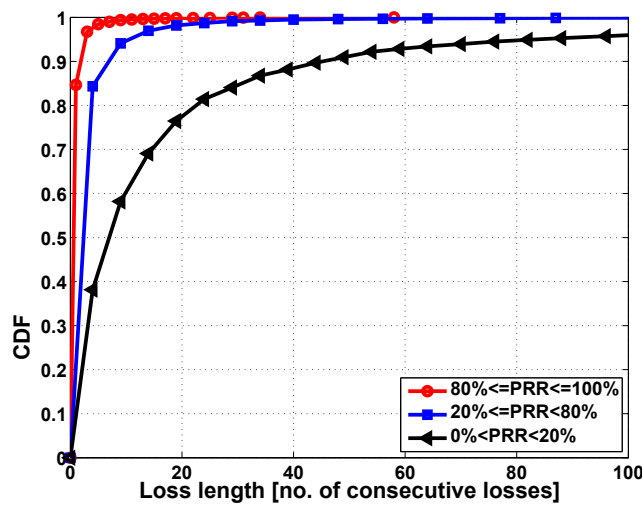

(a) Packet loss length.

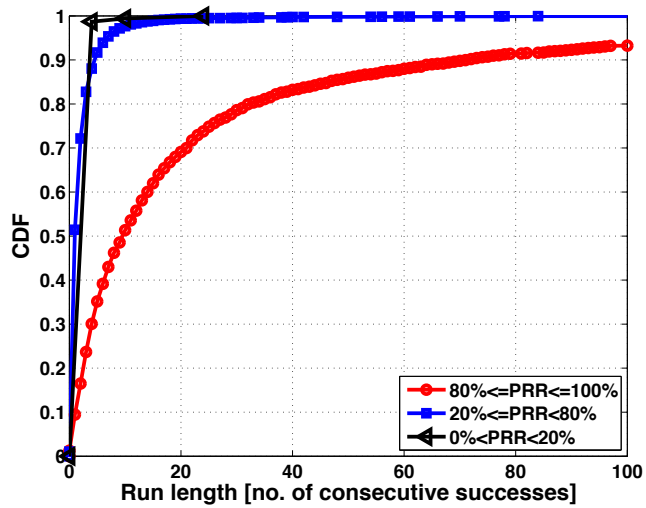

(b) Packet run length.

Fig. 2. Cumulative Distribution Function for each link category: good, intermediate, bad for a) packet loss length, b) packet run length, CC1101, 110B, 0dBm.

\section{Analysis of Packet Losses}

We call a run length the number of consecutive successful reception of packets and a loss length the number of consecutive lost packets.
Figure $2 \mathrm{a}$ shows that the distribution of packet losses and successful receptions depends on the link category. For instance, $98 \%$ of good links have an average 1.6 loss length, meaning that the probability of having consecutive lost packets is low. We have observed large average loss length of about 5.3 for intermediate links and about 56.8 for bad links, which shows that good links have independent losses with respect to intermediate and bad links. Yet, bad links may have bad states that last seconds. Figure $2 \mathrm{~b}$ illustrates that run length varies in function of link category. Good links stick to the good state for long periods, while bad links have quite small run length about 1 , which means that after only one successful reception bad links most probably return to the bad state.

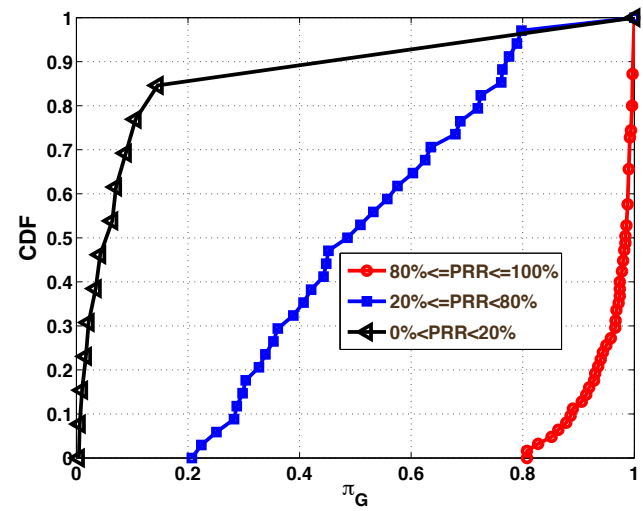

(a) Good state stationary probability $\pi_{G}$.

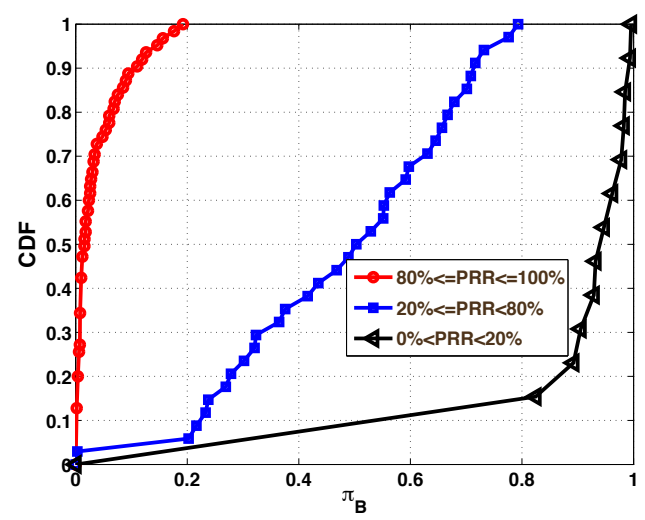

(b) Bad state stationary probability $\pi_{B}$

Fig. 3. Cumulative Distribution Function for each link category: good, intermediate, bad for a) stationary probability in good state $\pi_{G}$, b) stationary probability in bad state $\pi_{B}, \mathrm{CC} 1101,110 \mathrm{~B}, 0 \mathrm{dBm}$.

Figures $3 \mathrm{c}$ and $3 \mathrm{~d}$ show that good and bad stationary probabilities can discriminate quite well link categories.

TABLE V. GE PARAMETERs, 110B PACKeT SIZE, CC1101.

\begin{tabular}{|r|r|r|r|r|}
\hline Category & run length & loss length & $p$ & $r$ \\
\hline$P R R \geq 80 \%$ & 74.2 & 1.6 & 0.03 & 0.84 \\
\hline $20 \% \leq P R R<80 \%$ & 14.8 & 12.5 & 0.43 & 0.42 \\
\hline $0<P R R<20 \%$ & 2.2 & 43.4 & 0.81 & 0.07 \\
\hline
\end{tabular}

TABLE VI. GE PARAMETERS, 110B PACKET SIZE, CC1101.

\begin{tabular}{|r|c|c|r|r|}
\hline Category & $\pi_{G}$ & $\pi_{B}$ & $\pi_{\text {Loss }}$ & $\mu$ \\
\hline$P R R \geq 80 \%$ & 0.96 & 0.03 & 0.03 & 0.13 \\
\hline $20 \% \leq P R R<80 \%$ & 0.49 & 0.50 & 0.50 & 0.15 \\
\hline $0<P R R<20 \%$ & 0.07 & 0.92 & 0.92 & 0.12 \\
\hline
\end{tabular}


Tables V and VI summarize the parameters of the GE model: transition probabilities and stationary state probabilities. We can see that a good link has high $\pi_{G}(0.96)$ and low $\pi_{B}(0.03)$.

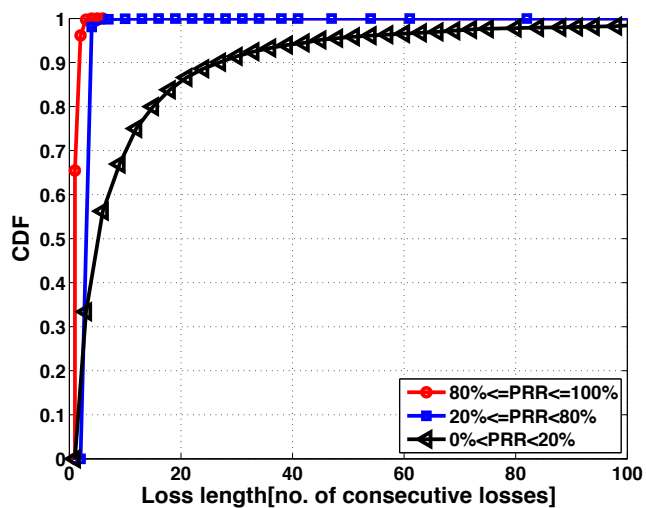

(a) Packet loss length.

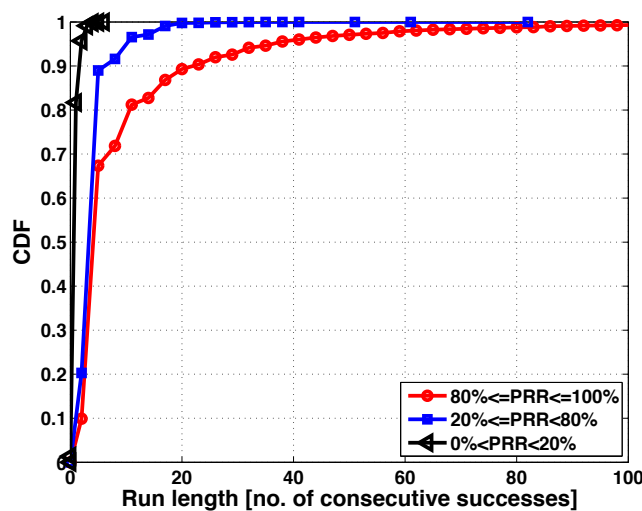

(b) Packet run length.

Fig. 4. Cumulative Distribution Function for each link category: good, intermediate, bad for a) packet loss length, b) packet run length, CC2420, $110 \mathrm{~B}, 0 \mathrm{dBm}$.

On the $\mathrm{CC} 2420$ radio platform, we have observed that loss length is below 1.5 for good links and increases for the decreasing link quality (cf. Figure 4a). Run lengths continue to be low for bad links (about 1) and high for good links (above 5) (cf. Figure 4b).

Again, $\pi_{G}$ discriminates links categories fairly well as it takes values within: $0.8-1$ (good links), $0.6-0.8$ (intermediate links), and $0-0.2$ (bad links) (cf. Figure 5a). Figure $5 \mathrm{~b}$ also shows that $\pi_{B}$ distinguishes well link categories $(0-0.2$ for good links, 0.2-0.4 for intermediate links, and 0.8-1 for bad links).

TABLE VII. GE PARAMETERS, 110B PACKETS, 0DBM, CC2420.

\begin{tabular}{|r|r|r|r|r|}
\hline Category & run length & loss length & $p$ & $r$ \\
\hline$P R R \geq 80 \%$ & 13.1 & 1.2 & 0.13 & 0.84 \\
\hline $20 \% \leq P R R<80 \%$ & 3.5 & 1.9 & 0.29 & 0.78 \\
\hline $0<P R R<20 \%$ & 1.0 & 28.0 & 0.92 & 0.08 \\
\hline
\end{tabular}

For the CC2420 radio, Table VII highlights that like for CC1101, good links have a small average loss length per link of 1.2. Intermediate links may encounter losses with an average length of 1.9 and bad links show a loss length of 28. Again, transition probabilities strongly depend on the link category:

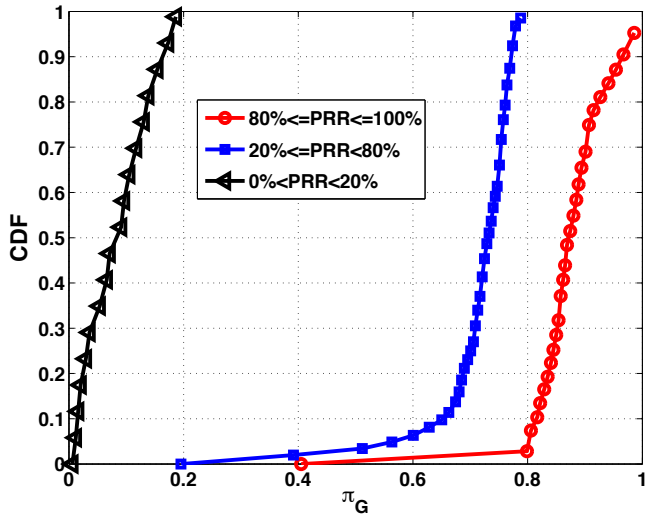

(a) Good state stationary probability $\pi_{G}$.

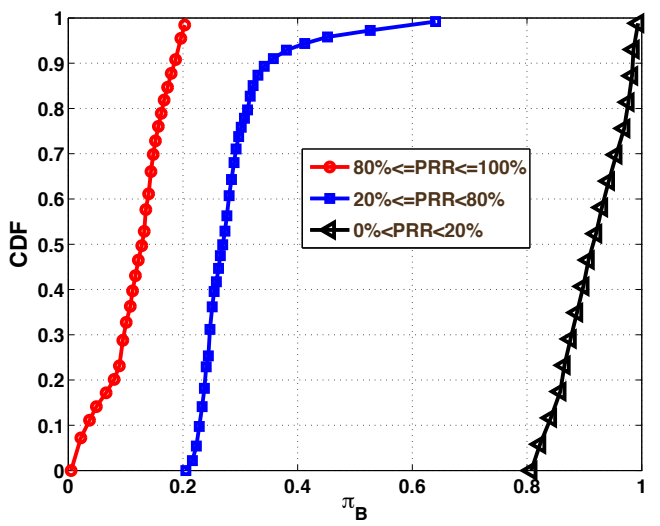

(b) Bad state stationary probability $\pi_{B}$.

Fig. 5. Cumulative Distribution Function for each link category: good, intermediate, bad for a) stationary probability in good state $\pi_{G}$, b) stationary probability in bad state $\pi_{B}, \mathrm{CC} 2420,110 \mathrm{~B}, 0 \mathrm{dBm}$.

TABLE VIII. GE PARAMETERS, 110B PACKETS, ODBM, CC2420.

\begin{tabular}{|r|l|l|r|r|}
\hline Category & $\pi_{G}$ & $\pi_{B}$ & $\pi_{\text {Loss }}$ & $\mu$ \\
\hline$P R R \geq 80 \%$ & 0.86 & 0.13 & 0.13 & 0.03 \\
\hline $20 \% \leq P R R<80 \%$ & 0.72 & 0.27 & 0.27 & -0.07 \\
\hline $0<P R R<20 \%$ & 0.08 & 0.92 & 0.92 & 0 \\
\hline
\end{tabular}

$p \sim 0.10$ for good links, $p \sim 0.3$ for intermediate, and $p \sim 0.9$ for bad links. On the other hand, $r$ decreases from $r \sim 0.80$ for good links to $r \sim 0.04$ for bad links. Table VIII shows that for CC2420, loss probability remains a good link quality discriminator $(0.13$ for good links, 0.27 for intermediate links, and 0.92 for bad links).

\section{Estimating PRR using LQI}

The results show that stationary probabilities $\left(\pi_{G}\right.$ and $\left.\pi_{B}\right)$ for the reception process are good discriminators of the link quality. In this section, we apply the GE model to the average (avg) of the LQI measured values for highly variable links.

We consider the avg LQI values of the received packets as a sequence of bits:

$$
f(\overline{l q i})= \begin{cases}1, & \text { if } \overline{l q i} \leq \overline{L Q I_{\text {threshold }}} \\ 0, & \text { otherwise }\end{cases}
$$

where $\overline{L Q I_{\text {threshold }}}$ is the threshold value of avg LQI that may be chosen with respect to the hardware and a given 
environment. For our analysis, we have chosen $\overline{L Q I_{\text {threshold }}}$ of 1.7: an avg LQI value below 1.7 corresponds to 1 (good reception) whereas an avg LQI above 1.7 corresponds to 0 (bad reception) (cf. Eq. 4). The threshold value of 1.7 was decided by observing fitting the LQI values with a Fermi-Dirac function [3].

For applying this approach, we have chosen a node that has only intermediate links in its neighborhood (Node 6, CC1101, OdBm) with different levels of PRR: $6 \rightarrow 20$ (PRR=64\%), $6 \rightarrow 57(\mathrm{PRR}=54 \%), 6 \rightarrow 34(\mathrm{PRR}=34 \%)$. We compute the stationary probability $\pi_{G}$ associated with the avg LQI to see if it can discriminate the quality of these links.

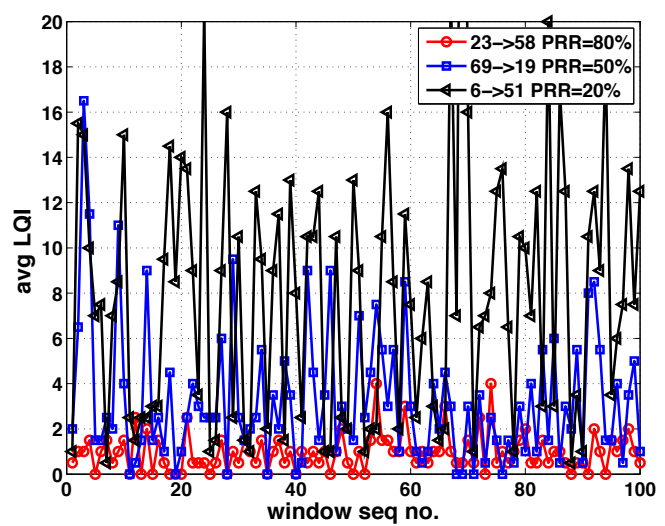

Fig. 6. Temporal evolution of avg LQI computed over windows of size 2, for link $23 \rightarrow 58(\mathrm{PRR}=80 \%)$, link $69 \rightarrow 19$ (PRR=50\%), and link $6 \rightarrow 51$ $(\mathrm{PRR}=20 \%), \mathrm{w}=2, \mathrm{CC} 1101,0 \mathrm{dBm}$.

Figure 6 depicts the temporal evolution of avg LQI computed over small windows of size 2 . We can observe important overlapping of the curves for the link with $\mathrm{PRR}=50 \%$ and PRR $=20 \%$.

To apply the GE model, we associate each value of the avg LQI with a sequence of bits and then, we compute the stationary probability $\pi_{G}$ for each sequence of five values as illustrated in Figure 7 (the figure presents the computation process for the average and standard variation of the LQI values, however, we only consider the average values of LQI due to the lack of space).

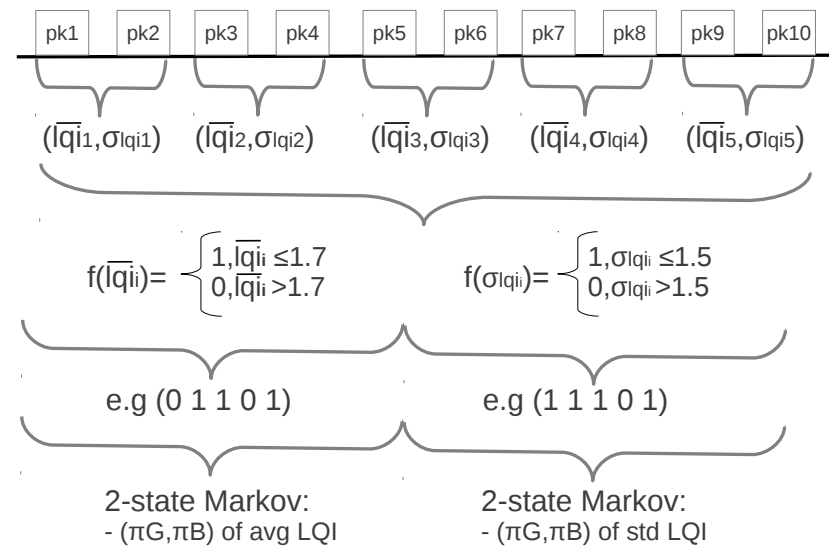

Fig. 7. Computing the stationary probabilities from avg and std LQI values.

Figure 8 shows stationary probability $\pi_{G}$ computed for avg LQI. We can see that the link with PRR of $80 \%$ can be easily distinguished from links with $50 \%$ or $20 \%$ of PRR $-\pi_{G}$ of avg LQI helps to discriminate the links with these values of PRR (cf. Figure 8).

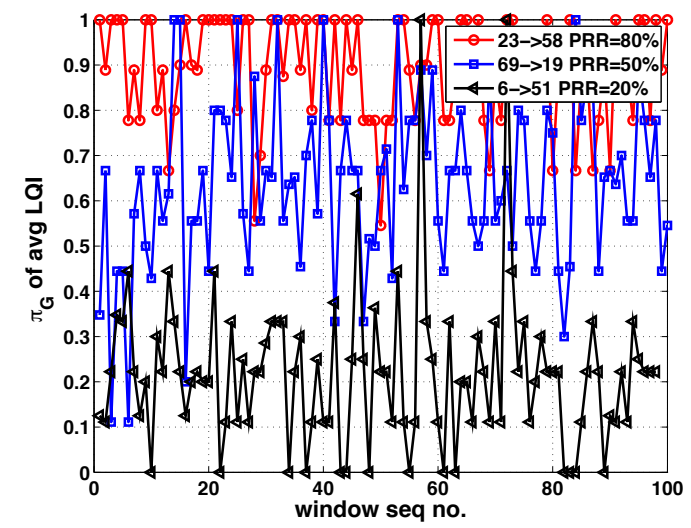

Fig. 8. Stationary probability $\pi_{G}$ derived from the measured avg LQI values (threshold of 1.7) for link $23 \rightarrow 58$ ( $P R R=80 \%)$, link $69 \rightarrow 19$ (PRR=50\%), and link $6 \rightarrow 51(\mathrm{PRR}=20 \%), \mathrm{w}=10, \mathrm{CC} 1101,0 \mathrm{dBm}$

Figure 9 shows that $\pi_{G}$ of avg LQI also enables fairly good differentiation between the links of PRR $=64 \%, P R R=54 \%$, and $\mathrm{PRR}=34 \%$.

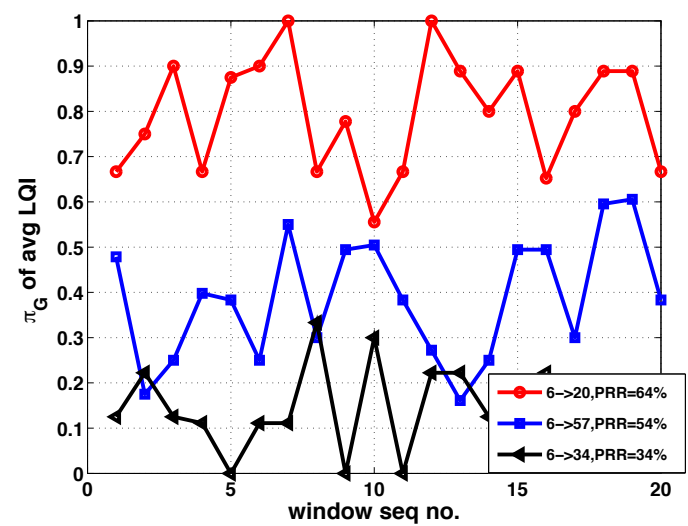

Fig. 9. Stationary probability $\pi_{G}$ derived from the measured avg LQI values (threshold of 1.7) for link $6 \rightarrow 20(\mathrm{PRR}=64 \%)$, link $6 \rightarrow 57(\mathrm{PRR}=54 \%)$, and link $6 \rightarrow 34(\mathrm{PRR}=34 \%), \mathrm{CC} 1101,0 \mathrm{dBm}$.

We have also evaluated the relevance and the efficiency of using stationary probabilities to categorize high variable CC2420 links. Like CC1101, we assess the temporal fluctuation of intermediate links with different reception ratio: $174 \rightarrow 127(\mathrm{PRR}=60 \%), 99 \rightarrow 179(\mathrm{PRR}=40 \%), 188 \rightarrow 105$ $(\mathrm{PRR}=20 \%)$. We apply the same approach as represented in Figure 7 with $\overline{L Q I_{\text {threshold }}}=70$.

Figure 10 shows that $\pi_{G}$ of avg LQI can very well distinguish among links with $\mathrm{PRR}=60 \%, \mathrm{PRR}=40 \%$, and $\mathrm{PRR}=20 \%$.

\section{RELATED WORK}

Much research work considered the problem of characterizing the quality of wireless links in sensor networks. On a 60 mote indoor/outdoor testbed, Zhao et al. [11] showed the existence of spatial gray regions corresponding to high variation in packet reception. Woo et al. [12] observed good connectivity of links to nodes up to $3 \mathrm{~m}$ and a transitional 


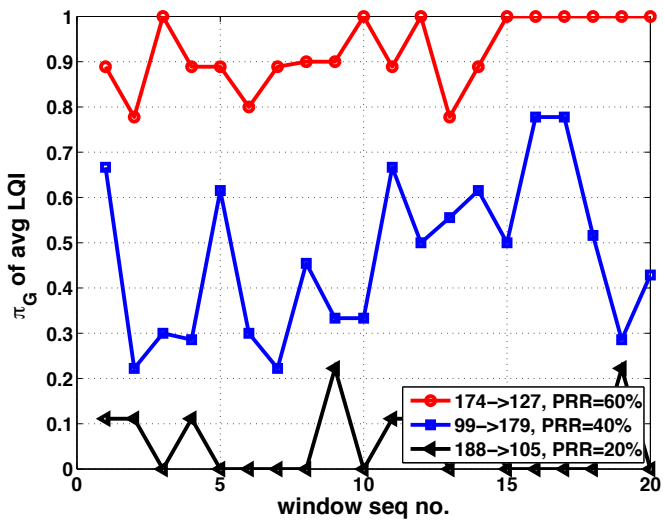

Fig. 10. Stationary probability $\pi_{G}$ derived from the measured avg LQI values (threshold of 70) for $174 \rightarrow 127$ (PRR=60\%), $99 \rightarrow 179$ (PRR=40\%), $188 \rightarrow 105(\mathrm{PRR}=20 \%), \mathrm{CC} 2420,0 \mathrm{dBm}$

(grey) zone between $3 \mathrm{~m}$ and $12 \mathrm{~m}$ with links exhibiting large variability. In another paper, the authors modeled packet loss with a binomial distribution and observed that good links are symmetric [13]. Other researchers confirmed the existence of three zones: the well connected, the transitional (grey), and the disconnected one [14], [15]. They reported that the links in the connected zone are stable contrary to the transitional zone.

As mentioned in the introduction, Srinivasan et al. [16] analyzed RSSI and LQI of CC2420. They highlighted the utility of RSSI in detecting links with good PRR and observed good correlation of LQI and PRR. Meier et al. [17] analyzed measurements of an indoor sensor network with CC2420 exhibiting high link quality variability and considered metrics derived from modeling the loss process as a Bernoulli process.

Tang et al. [18] evaluated the temporal and spatial link fluctuation in a factory environment and characterized channel variations. Bas et al. [19] demonstrated that the angle of the direction influences the correlation of the link quality with the distance. Rondinone et al. [20] analyzed the link quality in sensor networks and proposed to use as a link quality indicator the product of PRR and the normalized average RSSI.

\section{CONCLUSION}

In this paper, we have applied the Gilbert-Elliot model to analyze PRR based on measurements on a large-scale wireless sensor testbed. First, we derive the stationary probabilities of the Gilbert-Elliot model from the packet loss process. We show that the probabilities and other parameters such as run and loss lengths can very well discriminate between the main categories of link quality. Second, we also apply the same approach to LQI by transforming the average of LQI values to a $0-1$ process. Similarly, the Gilbert-Elliot probabilities of the avg LQI lead to good discrimination of quality for intermediate links. In the future, we plan to use the reported results to derive metrics for RPL and LOADng routing protocols and study their preformance on the IoT-LAB testbed.

\section{ACKNOWLEDGEMENT}

This work was partially supported by the French National Research Agency (ANR) IRIS project under contract ANR-11-
INFR-016, DataTweet project under contract ANR-13-INFR0008-01, and the European Commission FP7 CALIPSO project under contract 288879 .

\section{REFERENCES}

[1] T. Winter et al., "RPL: IPv6 Routing Protocol for Low power and Lossy Networks," IETF, RFC 6550, March 2012.

[2] T. Clausen et al., "The Lightweight On-demand Ad hoc Distancevector Routing Protocol - Next Generation (LOADng)," IETF, Work in Progress draft-clausen-lln-loadng-08, January 2013.

[3] A. Bildea, O. Alphand, F. Rousseau, and A. Duda, "Link Quality Metrics in Large Scale Indoor Wireless Sensor Networks," in Proc. of IEEE PIMRC, 2013.

[4] TI, "Calculation and Usage of LQI and RSSI." [Online]. Available: \{http://e2e.ti.com/support/low_power_rf/w/design_ notes/calculation-and-usage-of-lqi-and-rssi.aspx $\}$

[5] C. Burin des Rosiers et al., "SensLAB: Very Large Scale Open Wireless Sensor Network Testbed," in Proc. 7th TridentCOM Conference, Shanghai, Chine, Apr. 2011.

[6] G. Papadopoulos et al., "Adding Value to WSN Simulation Using the IoT-LAB Experimental Platform," in Proc. of IEEE WiMob, Lyon, France, October 7-9, 2013.

[7] T. Watteyne, S. Lanzisera, A. Mehta, and K. S. J. Pister, "Mitigating Multipath Fading through Channel Hopping in Wireless Sensor Networks," in Proc. of IEEE ICC, May 2010, pp. 1-5.

[8] K. Srinivasan, P. Dutta, A. Tavakoli, and P. Levis, "An Empirical Study of Low-Power Wireless," ACM Trans. Sen. Netw., vol. 6, no. 2, pp. 16:1-16:49, Mar. 2010

[9] E. N. Gilbert, "Capacity of a Burst-Noise Channel," vol. 39, Sep. 1960, pp. 1253-1265.

[10] G. Hasslinger and O. Hohlfeld, "The Gilbert-Elliott Model for Packet Loss in Real Time Services on the Internet," in 14th GI/ITG Conference on Measuring, Modelling and Evaluation of Computer and Communication Systems, 2008, pp. 1-15.

[11] J. Zhao and R. Govindan, "Understanding Packet Delivery Performance in Dense Wireless Sensor Networks," in Proc. SenSys, 2003, pp. 1-13.

[12] A. Woo, T. Tong, and D. Culler, "Taming the Underlying Challenges of Reliable Multihop Routing in Sensor Networks," in In SenSys. ACM Press, 2003, pp. 14-27.

[13] A. Woo and D. Culler, "Evaluation of Efficient Link Reliability Estimators for Low-Power Wireless Networks," EECS Department, University of California, Berkeley, Tech. Rep. UCB/CSD-03-1270, 2003.

[14] M. Zennaro, H. Ntareme, and A. Bagula, "Experimental Evaluation of Temporal and Energy Characteristics of an Outdoor Sensor Network," Proceedings of the Mobility '08 Conference, 2008.

[15] N. Baccour et al., "RadiaLE: A Framework for Designing and Assessing Link Quality Estimators in Wireless Sensor Networks," Ad Hoc Netw., vol. 9, no. 7, pp. 1165-1185, Sep. 2011.

[16] K. Srinivasan and P. Levis, "RSSI is Under Appreciated," in Proc. of the Third Workshop on Embedded Networked Sensors (EmNets), 2006.

[17] A. F. Meier, T. Rein, J. Beutel, and L. Thiele, "Coping with Unreliable Channels: Efficient Link Estimation for Low-Power Wireless Sensor Networks," in Proc. 5th Intl. Conf. Networked Sensing Systems (INSS 2008), Kanazawa, Japan, Jun 2008, pp. 19-26.

[18] L. Tang, K.-C. Wang, Y. Huang, and F. Gu, "Channel Characterization and Link Quality Assessment of IEEE 802.15.4-Compliant Radio for Factory Environments," IEEE Transactions on Industrial Informatics, vol. 3, no. 2, pp. $99-110$, May 2007.

[19] C. U. Bas and S. C. Ergen, "Spatio-Temporal Characteristics of Link Quality in Wireless Sensor Networks," in Proc. of WCNC, 2012, pp. 1152-1157.

[20] M. Rondinone, J. Ansari, J. Riihijärvi, and P. Mähönen, "Designing a Reliable and Stable Link Quality Metric for Wireless Sensor Networks," in Proceedings of the Workshop on Real-World Wireless Sensor Networks, ser. REALWSN'08, 2008, pp. 6-10. 